\title{
Provider documentation of patient education: a lean investigation ${ }^{*} \mathrm{C}$
}

\author{
Jean P. Shipman, MSLS, AHIP, FMLA; Erica W. Lake, MLS; Jessica Van Der Volgen, MLIS, AHIP; Darrin \\ Doman, MS, CCC-SLP
}

See end of article for authors' affiliations.

DOI: http://dx.doi.org/10.3163/1536-5050.104.2.012

Purpose: The study evaluates how providers give patient education materials and identifies improvements to comply with Meaningful Use (MU) requirements.

Methods: Thirty-eight patient-provider interactions in two health care outpatient clinics were observed.

Results: Providers do not uniformly know MU patient education requirements. Providers have individual preferences and find gaps in what is available. Accessing and documenting patient education varies among providers. Embedded electronic health record (EHR) materials, while available, have technical access barriers.

Conclusions: Providers' EHR skills and knowledge levels contribute to non-standardized patient education delivery.

Keywords: Patient Education as Topic, Meaningful Use, Electronic Health Records, Patient Compliance, Workflow

The provision of patient education at the point of care improves patient satisfaction and outcomes, improves quality of care, and lowers health care costs [1-3]. In this era of health reform and accountable care, patient education also affects Centers for Medicare and Medicaid Services (CMS) Meaningful Use (MU) reimbursement, as health care providers are required to fulfill patient and family engagement standards [4]. Ensuring that providers can access current patient-specific education material quickly and easily within the electronic health record (EHR) is increasingly important. Health sciences librarians frequently provide consumer health information and patient education, both of which contribute to improved health care quality, improved patient outcomes, and lowered health care costs. With their organizational skills and knowledge, librarians can contribute their expertise to the benefit

\footnotetext{
* Based on a presentation at MLA '15, the 115th Annual Meeting of the Medical Library Association; Austin, TX; May 19, 2015, which won 1st place for best research paper by MLA Research Section.

A supplemental appendix is available with the online version of this journal.
}

of "Lean" initiatives and teams at their institutions. This case study illustrates one such example of how a Lean team incorporated the skills and experience of three health sciences librarians.

\section{University of Utah Health Care}

University of Utah Health Care (UUHC) is the Intermountain West's only academic health care system, combining patient care, medical research, and education. The system provides care for the citizens of Utah and residents of 5 surrounding states in a referral area that encompasses more than $10 \%$ of the continental United States. UUHC comprises 4 hospitals, several specialty centers, and 11 neighborhood community clinics, offering primary care and specialty services along with pharmacy, lab, and X-ray services [5].

\section{Electronic health record}

UUHC maintains a wide-area network supporting information technology (IT) applications across the 
hospitals, specialty centers, and clinics, encompassing more than 6,000 clinical and administrative users. IT installs, configures, maintains, and provides training on the EHR in all patient environments. UUHC utilizes the Epic EHR, with training required for all users.

\section{Meaningful Use}

MU is the set of standards defined by CMS incentive programs that governs the use of EHRs and allows eligible providers and hospitals to earn incentive payments by meeting specific criteria. The goal of MU is to promote the adoption of EHRs to improve health care, as they offer many benefits for providers and patients, if used appropriately [6]. These benefits include complete and accurate patient information for providers, greater access to information for providers, and increased access to medical records and health information for patients.

MU includes both a core set and a menu set of objectives. The objectives are being implemented in stages, with each stage becoming increasingly stringent concerning patient education. Stage 1 menu set objectives focus on using the EHR to identify patient-specific education resources in an effort to engage patients and their families in their care. At the time of this study, this became a core objective with Stage 2. If an organization fails to demonstrate that all MU eligible providers are meeting the Stage 2 objectives, reduced CMS reimbursement rates result [6].

\section{Krames On-Demand}

Patients perceive written patient education information as particularly beneficial $[7,8]$. Krames StayWell is the largest US provider of patient education content in print. Krames On-Demand offers a database of educational content, including thousands of illustrated handouts and discharge instructions, and over 2,000 drug information handouts. Krames On-Demand directly integrates patient education content into the EHR workflow and is certified by the Office of the National Coordinator Authorized Testing and Certification Body to help meet MU requirements for electronic copies of discharge instructions, patient-specific education resources, and medication reconciliation. Prior to MU requirements, UUHC providers accessed patient education information only via the Krames On-Demand website.
Lean

Toyota Manufacturing developed the Lean process. Its overarching goal is to evaluate steps in a process to bring value to the customer by eliminating waste and improving efficiency. A key element is to reduce waste and unnecessary steps when possible to streamline or standardize processes.

In early 2013, the senior vice president for health sciences; dean, School of Medicine; and chief executive officer, University Health Care, initiated a program to encourage adoption of this process throughout the enterprise. She commissioned the School of Business Executive Education Program to educate cohorts of physicians and leaders about Lean in order for them to apply the process to health care issues. Fifteen individuals participated in the first cohort, forming teams to address health care issues. Teams had three months to receive training and frame and develop projects that addressed an appropriate problem statement.

The following describes the process used by one team to address patient education documentation by clinic staff to meet MU criteria. This team included individuals from the clinical education staff, information technology, and the health sciences library. Outside expertise was sought as needed to obtain further information. Teams were required to deliver the following: an A3 template, a professional publication, and a fifteen-minute summary presentation for UUHC leadership.

\section{METHODS}

\section{Assessment of the current situation}

A flow diagram of the perceived current state of patient education documentation was created based on the team's knowledge of outpatient encounters. Online only Figure 1 illustrates the high variability in patient education provision and documentation, with only some paths reliably leading to documentation that is valid for MU attestation. To ensure that outpatient clinics continue to meet these MU criteria, increasing the percentage of encounters that included appropriate patient education documentation by decreasing variability in the documentation process was necessary.

To ensure that the flow diagram reflected all process variability and to begin to look for causes of these differences, the team conducted a Gemba walk, 
a Lean practice. In a Gemba walk, the team visits the location where the process occurs and purposefully evaluates the flow and context.

\section{Data collection}

For the Gemba walk, 2 UUHC outpatient clinics were selected, representing a range of MU compliance for the patient education measure. In 2012, Clinic 1 (family medicine) provided and correctly documented patient education for $93 \%$ of unique patients. Clinic 2 (internal medicine and family practice) had a lower rate of documented patient education at $53 \%$.

To ensure the consistency of information gathered across observations, a shadowing tool recorded each patient-provider encounter. The observer used the tool to document who searched for and provided patient education, what type was provided, did documentation occur, and how easy was locating appropriate patient education materials (Appendix, online only). Each of the six team members shadowed a provider at both Clinic 1 and Clinic 2 for two to three hours at a time, over a two-week period in February 2013. Providers included physicians, medical residents, and nurse practitioners. To minimize the impact on regular patient education behavior, providers were informed that team members were observing the functionality of the EHR. In addition to shadowing, team members recorded relevant comments from providers and informal observations. The data were analyzed comparing the two clinics.

\section{RESULTS}

Table 1 summarizes the clinic observation data. Four main themes surfaced as to why variation occurs in documenting patient education across all patientprovider interactions, and these themes are discussed in the following paragraphs.

\section{Inconsistent processes for accessing and documenting patient education}

At UUHC, patient education types 1 and 2 are the only IT-designated workflows that comply with MU Stage 1 standards. Process types 3 through 5 can comply with MU Stage 1 if relevant information is copied or manually entered into a designated EHR field. If providers do not document the provision of patient education or document patient education outside this designated field, IT is unable to audit for MU compliance. In MU Stage 2, at the time of this study, only types 1 and 2 met requirements.

\section{Content gaps and preferences}

UUHC licenses Krames On-Demand content to embed into the EHR workflow. Providers are able to search by keyword and include relevant content in visit summaries in patient records or print a hard copy. Providers reported instances where desired topics were nonexistent, nonspecific, or not easily found. This resulted in providers using other resources; for example, web pages outside the EHR, preprinted reference materials, or their own developed materials.

\section{Technical search difficulties}

Providers and support staff identified inconsistencies in search functions between embedded and web versions of Krames On-Demand. For example, using the search term "pink eye" yielded results in the Krames On-Demand website but yielded "no results" when searched through Epic. IT confirmed programming anomalies between the two environments, even though there should be no difference.

\section{Variable provider awareness of Meaningful Use patient education requirements}

Team member interactions with providers led to a realization that there is a continuum of understanding of MU Stage 1 patient education requirements among providers, with few being aware of the more stringent MU Stage 2 requirements. Moreover, new-hire EHR training contained little information about patient education documentation workflow, and no information about MU requirements.

\section{DISCUSSION}

The team was surprised to discover that the majority of clinics had high compliance rates for MU Stage 1. Observations indicated a higher level of variability in how patient education was delivered than originally perceived. It was clear that few providers would meet Stage 2 requirements if they continued with the 


$\begin{array}{rr}4 & 10.5 \% \\ 11 & 28.9 \% \\ 3 & 7.8 \% \\ 4 & 10.5 \% \\ 14 & 36.8 \% \\ 1 & 2.6 \% \\ 1 & 2.6 \%\end{array}$

Note: In five patient-provider interactions, the type of patient education was double-coded, meaning providers offered two different modes of patient education. Only the first level of coding was included in this table.

Table 1

Patient education

same workflow processes. It is also unknown how frequently patient education is provided but not documented in a method that is auditable.

Some key differences in the workflow between the two study clinics were observed. One major difference was the use of scribes in searching for and documenting patient education. When used effectively, a support staff member (typically a medical assistant) was able to compile printed material and document patient education, while the provider offered verbal education to the patient. Another difference was convenient location and functionality of printers (i.e., printouts going to an unknown printer wasted provider time and caused frustration).

Challenges inherent in this project included the lack of governance to foster behavioral changes in the myriad number of individuals involved in patient education, such as representatives from IT, informatics, EHR support/trainers, hospital leadership, providers, nurses, clinic support staff, vendors, medical practice group, and department financial offices. Support for providing patient education differently needs endorsement by top administration.

Accurate figures reflecting physician salary or revenue generated by an average clinic visit were difficult to obtain to quantify the financial impact of this project's implementation. Moreover, due to the complexity of the MU reimbursement rules, isolating the exact cost associated with patient education compliance alone is not possible.

EHR users' skills and knowledge levels contribute to the variability of patient education workflow processes. Adequate support from IT, increased collaboration among vendors, relevant and userfriendly patient educational resources, and focused training serve to improve provider compliance with MU standards. Standardizing the documentation process reduces waste, adds value, and has a positive financial impact through cost avoidance, revenue generation, and MU compliance. Additional scrutiny of this issue will prove useful in future MU stages.

The four main patient education challenges as described above result in decreased workflow efficiencies, variability in how patients receive education products, provider frustration, increased risk of not meeting MU requirements for patient education, and financial impacts.

The following interventions or counter measures to improve patient education workflow efficiencies were initiated:

1. Implement Epic training on patient education for new employees that includes MU information regarding the financial impact of properly documenting patient education, as well as the preferred EHR workflow.

2. License an additional library of patient education materials, the Exit-Writer library produced by Krames StayWell.

3. Promote a standard process for developing custom content to fill Krames StayWell gaps. 4. Initiate and conduct regular meetings between UUHC IT, Epic, and Krames StayWell representatives to review technical issues.

5. Display a graphic in the clinics that depicts providers' MU compliance rates over time. This is a standard Lean technique that fosters "visual accountability."

This project's key financial impact is cost avoidance. By saving health providers' time, patient loads can be increased, which results in increased revenue. However, when this idea was suggested, the providers were less than enthusiastic. They 
expressed a desire to spend any "saved" time in providing additional patient education rather than increasing patient load. A calculation of annual clinic visits, multiplied by 1 minute of an average physician's salary, potentially generates a cost avoidance of $\$ 2,231,053$ annually. This same time savings, applied to additional patient visits averaging $\$ 200$ per visit for the 343 eligible providers, when multiplied by 225 working days per year, could produce $\$ 15,435,000$ in new revenue annually. Of course, if the UUHC meets the MU criteria, it receives income for doing so and consequently avoids any penalties.

\section{ACKNOWLEDGMENTS}

The authors acknowledge the other valuable members of their Lean Project Team: Madeline Araya, RN, MS, senior systems analyst, EpicCare Team/OneChart, and Alisha Richins, RN, BSN, then outpatient clinics nurse educator, University of Utah Health Care.

\section{REFERENCES}

1. Eckman MH, Wise R, Leonard AC, Dixon E, Burrows C, Khan F, Warm E. Impact of health literacy on outcomes and effectiveness of an educational intervention in patients with chronic diseases. Patient Educ Couns. 2012 May;87(2):143-51. DOI: http://dx.doi.org/10.1016/j.pec. 2011.07.020.

2. Joint Commission. Comprehensive accreditation manual for hospitals: the official handbook. The Commission; 2007. PC 21.

3. Larson CO, Nelson EC, Gustafson D, Batalden PB. The relationship between meeting patients' information needs and their satisfaction with hospital care and general health status outcomes. Int J Qual Health Care. 1996 Oct; 8(5):447-56.

4. Kreofsky L. Engaging staff to engage patients: patient engagement is essential for meaningful use, and studies show it is becoming more definitively linked to consumer satisfaction. Health Manag Technol. 2013 Feb;34(2):12-3. 5. University of Utah Health Care [Internet]. [cited 14 Jun 2013]. <http://healthcare.utah.edu $>$.

6. Centers for Medicare and Medicaid Services. Electronic health records (EHR) incentive programs [Internet]. The Centers [cited 14 Jun 2013]. < https://www.cms.gov/

Regulations-and-Guidance/Legislation/

EHRIncentivePrograms/>.
7. Felley C, Perneger TV, Goulet I, Rouillard C, Azar-Pey N, Dorta G, Hadengue A, Frossard JL. Combined written and oral information prior to gastrointestinal endoscopy compared with oral information alone: a randomized trial. BMC Gastroenterol. 2008 Jun 3;8:22. DOI: http://dx. doi.org/10.1186/1471-230X-8-22.

8. Sheard C, Garrud P. Evaluation of generic patient information: effects on health outcomes, knowledge and satisfaction. Patient Educ Couns. 2006 Apr;61(1):43-7.

\section{AUTHORS' AFFILIATIONS}

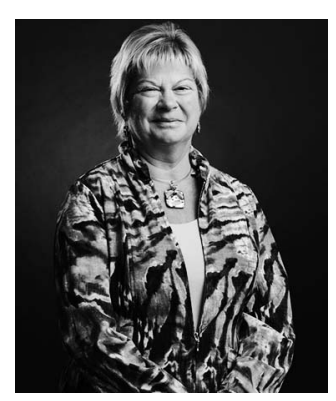

Jean P. Shipman, MSLS, AHIP, FMLA, jean.shipman@ utah.edu, Director, Spencer S. Eccles Health Sciences Library, National Network of Libraries of Medicine MidContinental Region, and National Library of Medicine Training Center, and Director for Information Transfer, Center for Medical Innovation; Erica Lake, MLS, erica.lake@utah.edu, Associate Director, Hope Fox Eccles Health Library; Jessica Van Der Volgen, MLIS, AHIP, j.vandervolgen@utah.edu, Assistant Director, National Network of Libraries of Medicine and National Library of Medicine Training Center; Spencer S. Eccles Health Sciences Library, 10 North 1900 East, Building 589, Salt Lake City, UT 84112; Darrin Doman, MS, CCC-SLP, Darrin.Doman@ hsc.utah.edu, Staff Development Educator, University of Utah Health Care, 50 North Medical Drive, HSEB 1100, Salt Lake City, UT

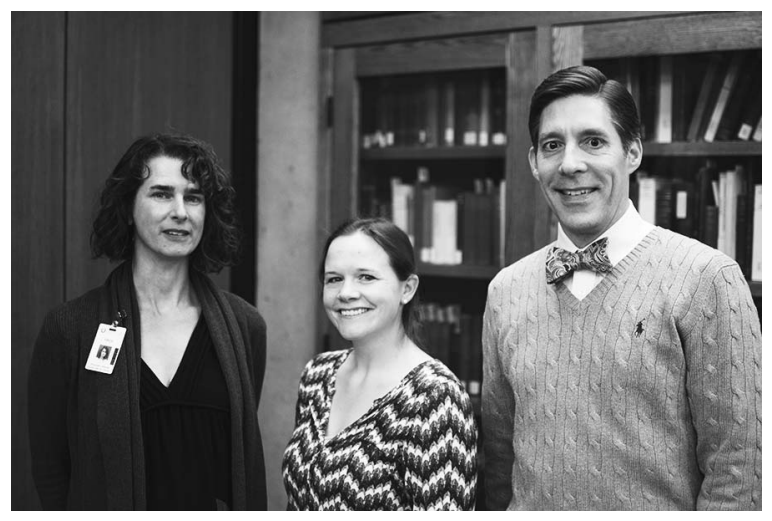

Received September 2015; accepted December 2015 